\title{
Instruments économiques et gestion de l'eau d'irrigation en France
}

\author{
par Marielle Montginoul, Thierry Rieu \\ CEMAGREF, Division Irrigation
}

\section{INTRODUCTION}

\subsection{L'eau comme bien économique}

La France est un pays où les ressources en eau sont abondantes naturellement ou du fait des aménagements réalisés notamment dans le sud de la France. Toutefois, des tensions sur l'usage de l'eau demeurent, en particulier en période d'étiage. Dans ces situations, l'eau devient rare et ne peut plus être considérée comme un bien libre et donc gratuit. Elle entre dans la catégorie des biens économiques. C'est aussi un bien collectif car il répond à différents usages et participe au maintien de la qualité et au fonctionnement des hydrosystèmes.

\begin{abstract}
1.2 Les usages de l'eau
L'estimation des prélèvements et les consommations nettes permettent de rendre compte de la répartition entre les différents usages (Tableau 1).

Les différentes consommations ont connu les évolutions suivantes:

- l'usage de l'eau potable a progressé régulièrement de 1 à 2\% par an et s'est stabilisé depuis 1990 ;

- la consommation de l'eau par l'industrie a fortement diminué, de $20 \%$, pendant les années 80 , du fait de la disparition d'industries traditionnelles fortement consommatrices d'eau et de l'effort de maîtrise énergétique ou de dépollution ;
\end{abstract}

\section{Economic instruments and imigation water management in France}

In spite of abundant reserves in France, imbalances between water needs and resources, conflicts between different categories of users are likely to appear during low river flow periods. In these situations, as water becomes a scarce resource, it can not be considered any longer as a free good but as an economic good. In a context of a limited water resource, community tries to induce farmers to minimise their water consumption by developing and implementing economic instruments such as water markets, rationing procedures (quota) or appropriate water pricing.

However, market mechanisms can not function efficiently without an appropriate legislative frameworl. In France, the laws of 1964 and 1962 and the European regulation define the principles of water management.

The water law of 1992 insists on the uniqueness of water resource, imposes measuring devices and defines negotiation areas. The implementation of this law renew the interest for incentive economic instruments, the conditions required for their implementation and their limitations.

In France, water pricing and quota are implemented. These instruments are not incompatible and are likely to complenent each other:

One manager (Compagnie du Bas-Rhôle-Languedoc) particularly takes into account water demand, ano. ther (Compagnie d'Amenagenent des Coteaux de Gascogne) applies a system combining quota and water pricing, the last (Societe du Canal de Provence) uses marginal cost pricing. 
Tableau 1. - Prélèvements et consommations de la ressource en eau selon les usages.

\begin{tabular}{|l|c|c|c|r|}
\hline \multirow{2}{*}{ Usages de l'eau } & \multicolumn{2}{|c|}{ Prélèvements } & \multicolumn{2}{|c|}{ Consommations nettes } \\
\cline { 2 - 5 } & Milliards de $\mathrm{m}^{3}$ & $\%$ du total & Milliards de $\mathrm{m}^{3}$ & $\%$ du total \\
\hline Eau potable & 6,1 & 16,18 & 2,5 & 43,86 \\
Industries non raccordées à un réseau & 4,4 & 11,67 & 0,4 & 7,02 \\
Centrales thermiques & 22,3 & 59,15 & 0,4 & 7,02 \\
Agriculture & 4,9 & 13,00 & 2,4 & 42,10 \\
\hline Total & 37,7 & 100 & 5,7 & 100 \\
\hline
\end{tabular}

Source: Ministère de l'environnement (données de 1990).

- la consommation en eau des centrales thermiques est stable, voire en diminution ;

- la consommation de l'eau pour l'irrigation est importante. L'irrigation s'est développée en France depuis des siècles dans les régions déficitaires en eau situées dans le sud-est et le sud-ouest de la France. En 1970, l'irrigation concernait surtout les plaines du sud-est et du sud-ouest et une partie des vallées de la Loire moyenne. Entre 1975 et 1988 , il y a eu une forte augmentation des volumes prélevés $(+43 \%)$, ce qui est dû au développement des surfaces irriguées dans les zones traditionnelles de l'irrigation mais aussi dans les régions du centre et de l'ouest de la France.

On assiste à une situation très diversifiée dans les bassins : au nord et au nord-est, l'irrigation occupe une part modeste : ailleurs, elle représente 43 à $66 \%$ du total de l'eau consommée en 1990 , voire plus de $80 \%$ en période estivale.

\subsection{Les acteurs de la gestion de l'eau}

On peut schématiser la gestion de l'eau d'irrigation en France par quatre acteurs : la collectivité (Etat, collectivités locales), l'agence de l'eau, le gestionnaire et l'agriculteur. Leurs objectifs diffèrent et pourtant nécessitent d'être pris en compte simultanément.

- La collectivité cherche à maximiser le bien-être des individus en tenant compte d'un souci d'équité, d'une alimentation suffisante en eau potable et en minimisant le coût de l'incitation. Elle a en charge la police des eaux.

- L'agence de l'eau incite à gérer de manière optimale la ressource en eau en luttant contre la pollution, en améliorant les ressources en quantité et en qualité, en incitant les acteurs concernés par l'eau à se concerter. Elle a un objectif d'équilibre durable du système.

- Le gestionnaire est un organisme chargé de développer et de gérer les ouvrages permettant de desservir les usagers. Ses objectifs sont liés à la mission qui lui est confiée et diffèrent suivant que le gestionnaire a vocation à satisfaire les

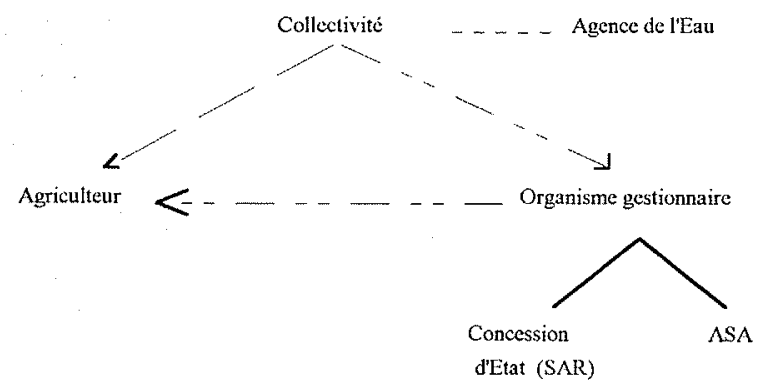

1. Schéma simplifié des relations entre les différents acteurs intervenant dans la gestion de l'eau. besoins en eau de toute nature de la collectivité (à travers les concessions d'Etat, pour les grands périmètres) ou seulement les besoins en eau d'irrigation (comme les Associations Syndicales Autorisées, pour les périmètres de quelques centaines d'hectares). Les modalités de gestion dépendent de son statut. L'objectif minimum est d'équilibrer le budget et de maintenir le périmètre irrigué.

- Les agriculteurs visent à satisfaire des objectifs d'entreprise (comme maximiser le profit, minimiser les coûts de production ou minimiser le risque de production) et de ménage (revenu minimum, par exemple).

Dans les systèmes de production irriguée, on observe une valorisation très différente de l'eau en raison de l'hétérogénéité de comportement des agriculteurs. Dans tous les pays, la diversité des pratiques d'irrigation doit être prise en compte pour prévenir les comportements opportunistes et pour préserver l'équité entre agriculteurs face à l'eau.

Après une description du cadre dans lequel se situe la gestion de l'eau d'irrigation en France, le rapport précise les modalités d'application des instruments économiques (tarification, quotas, marchés de l'eau) à partir de l'analyse de quatre cas concrets en France.

\section{UN CADRE LÉGISLATIF ET ADMI- NISTRATIF NÉCESSAIRE À LA MISE EN PLACE D'INSTRUMENTS ÉCO- NOMIQUES}

Dans le cadre d'une ressource en eau limitée, la collectivité cherche à inciter les agriculteurs à minimiser les prélèvements d'eau, ce qui peut être fait par la mise en place d'instruments économiques comme les marchés de l'eau, de quotas ou d'un système tarifaire adéquat.

La législation apparaît toutefois nécessaire pour permettre aux mécanismes de marché de fonctionner efficacement. Dans le domaine de l'eau, ce sont les lois françaises de 1964 et 1992 qui encadrent la gestion de l'eau d'irrigation, ainsi que des réglementations européennes.

1) Proclamation de l'unité de la ressource en eau (eau souterraine, eau de surface ou eau de la mer dans la limite des eaux territoriales).

2) Création des comités de bassin et des agences de l'eau (organismes de concertation et de gestion dans les six bassins hydrographiques français) pour améliorer les ressources en quantité et en qualité et lutter contre la pollution. Les agences de l'eau sont chargées d'appliquer les principes pollueur-payeur et préleveur-payeur. Par la perception de redevances sur les utilisateurs de l'eau du bassin, elles disposent de fonds qu'elles utilisent pour participer à la réalisation d'investissements en attribuant des subventions et/ou en accordant des prêts. 
Trois dispositions tempèrent l'application de ces principes :

- l'instrument économique s'accompagne d'une action réglementaire,

- le montant des redevances est voté par des instances où les redevables sont représentés,

- un mécanisme de financement est instauré au niveau du bassin versant.

Dans la pratique, on peut regretter que la fonction d'incitation économique n'ait pas été davantage développée.

3) La loi sur l'eau du 3 janvier 1992: une gestion équilibrée de la ressource en eau.

A cette fin, elle envisage :

- la préservation des systèmes aquatiques,

- la protection contre toute pollution (dont la pollution diffuse),

- le développement et la protection de la ressource,

- la valorisation de l'eau en tant que ressource économique.

Pour tendre vers une gestion équilibrée de la ressource en eau, elle crée, au niveau d'un bassin versant, des SDAGE (Schéma Directeur d'Aménagement et de Gestion des Eaux) dans l'optique d'une planification des aménagements, et, au niveau d'une unité hydrographique ou d'un système aquifère, des SAGE (Schéma d'Aménagement et de Gestion des Eaux) dans une gestion plus opérationnelle et plus décentralisée. Une structure à géométrie variable, la Commission Locale de l'Eau (CLE), où sont représentés notamment tous les usagers de l'eau et les collectivités concernées, est mise en place et est conçue comme un lieu de gestion locale des usages. Les SDAGE sont obligatoires dans les six grands bassins hydrographiques français. L'instauration de SAGE s'avérant complexe, il est prévu de les mettre en œuvre progressivement et en priorité dans les zones sensibles où les problèmes (pollution, conflits d'usages) sont les plus aigus.

Elle rend obligatoire l'installation de moyens de mesures ou d'évaluation appropriés, et interdit la pratique du forfait, sauf exceptions dûment justifiées, pour la tarification de l'eau potable au profit d'une tarification binôme. Pour les autres usages de l'eau, le forfait n'est pas interdit.

Nous allons étudier les principes d'application des instruments économiques avant de décrire le cas français.

\section{PRINCIPE ET NATURE DES INS- TRUMENTS DE GESTION DE L'EAU D'IRRIGATION}

Trois instruments économiques peuvent être envisagés pour gérer l'eau d'irrigation: la tarification, les quotas ou les marchés de l'eau.

\subsection{La tarification}

Pour qu'une tarification soit efficace, certaines conditions doivent être remplies.

\section{I.I Conditions nécessaires}

1) Une sensibilité de la demande par rapport au prix de l'eau.

Les agriculteurs doivent être sensibles au prix de l'eau: lorsque le prix de l'eau change, les agriculteurs réagissent en terme d'assolement, de conduite d'irrigation et par la suite d'apports d'eau à la plante. Un point de référence communément utilisé est l'ETM (Evapo-Transpiration Maximale) qui représente l'optimum agronomique.

Ainsi, trois cas peuvent être distingués où la tarification est inefficace pour inciter les agriculteurs à économiser de l'eau :
- le cas de cultures à forte valeur ajoutée : l'élasticité de la demande en eau par rapport au prix est alors très faible ou nulle. Dans ce cas-là, une augmentation du prix de l'eau ne modifie pas la consommation d'eau d'imigation,

- le cas d'exploitations en situation de survie: le fait de faire payer l'eau peut alors les contraindre à ne plus irriguer ou à disparaitre dans les cas extrêmes,

- le cas où il n'y a pas d'alternative à la culture en sec ou quand l'agriculteur ne peut pas diminuer sa consommation d'eau sans une forte perte de rendement.

2) Un système tarifaire facilement compréhensible.

Il doit être facilement compris par les consommateurs d'eau : cela exclut donc les systèmes complexes, comme par exemple plusieurs tarifs avec des paliers différents, qui peuvent dérouter les utilisateurs et leur transmettre des signaux erronés.

3) Un système tarifaire stable dans le temps ou à des horizons compatibles avec les prises de décision des agriculteurs.

4) Un système peu coûteux à mettre en place pour le gestionnaire.

L'élasticité de la demande par rapport au prix de l'eau est en étroite corrélation avec la rentabilité privée de l'eau d'irrigation. Celle-ci est fortement variable selon au moins quatre critères:

- la situation géographique de l'exploitation (climat, sols),

- les caractéristiques de la ressource en eau,

- l'orientation de production et les conditions de marché associées,

- les caractéristiques de l'exploitation.

Il en ressort qu'une réponse générale ne peut être proposée d'un point de vue privé et probablement aussi d'un point de vue collectif.

Ainsi, pour les cultures maraîchères et arboricoles, la rentabilité privée est généralement élevée compte tenu de la valeur ajoutée. De plus, le prix de l'eau représente une faible part (de l'ordre de 2 à $4 \%$ ) des charges totales de ces exploitations. C'est pourquoi, jusqu'à un certain prix, la fonction de demande en eau révèle une rigidité au prix de l'eau d'irrigation comme le montre la fonction de demande établie pour des exploitations orientées vers l'arboriculture et le maraîchage de plein champ. Mais la demande d'eau est limitée par les contraintes que l'introduction de l'irrigation imposent aux exploitations : risques de marché, trésorerie, embauche de main d'œuvre, ainsi que par les stratégies de production des exploitants.

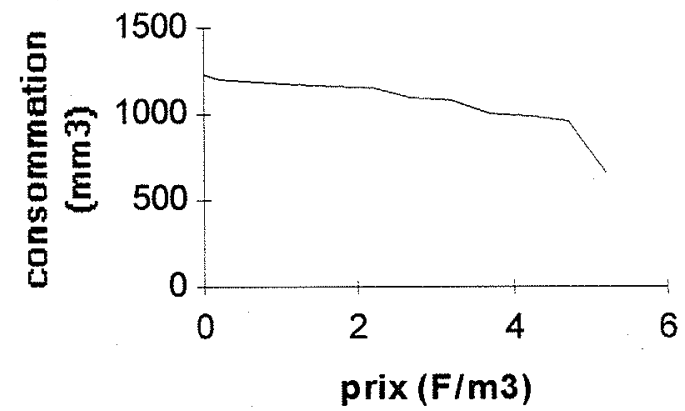

2. Courbe de demande en eau. Source : Rieu, Gleyses, Houille Blanche, ${ }^{\circ}$ 2-3, 1993.

\section{I.2 Conditions suffisantes}

1) La possibilité de contraindre les demandeurs à payer l'eau qu'ils utilisent.

2) La tarification doit refléter le coût marginal.

Elle doit être un indicateur de rareté et d'utilité donc un instrument de rationnement pour éviter des conflits d'usages, 
Tableau 2. - Efficacité des modes de gestion en fonction de la sensibilité par rapport au prix.

\begin{tabular}{|l|l|l|l|l|}
\hline $\begin{array}{c}\text { Sensibilité de la } \\
\text { la consommation }\end{array}$ & $\begin{array}{l}\text { Sensibilité } \\
\text { du revenu }\end{array}$ & $\begin{array}{c}\text { Tarification binôme } \\
\text { simple }\end{array}$ & \multicolumn{1}{|c|}{$\begin{array}{c}\text { Tarification binôme } \\
\text { par paliers }\end{array}$} & \multicolumn{1}{c|}{ Quotas } \\
\hline Faible & Faible & $\begin{array}{l}\text { C'est uniquement un } \\
\text { moyen de recouvre- } \\
\text { ment des frais engagés }\end{array}$ & $\begin{array}{l}\text { C'est uniquement un moyen de } \\
\text { recouvrement des frais engagés }\end{array}$ & $\begin{array}{l}\text { C'est la politique qui } \\
\text { semble la plus adaptée }\end{array}$ \\
\cline { 2 - 5 } & Forte & $\begin{array}{l}\text { Non, aucune incitation } \\
\text { et trop de consé- } \\
\text { quences sociales }\end{array}$ & Envisageable, mais pas efficace & $\begin{array}{l}\text { C'est la politique qui } \\
\text { semble la plus adaptée }\end{array}$ \\
\hline Forte & Faible & $\begin{array}{l}\text { Elle peut être appliquée } \\
\text { et donne de bons résul- } \\
\text { tats en terme d'incita- } \\
\text { tion à l'économie d'eau }\end{array}$ & $\begin{array}{l}\text { Idem que la politique précé- } \\
\text { dente }\end{array}$ & Envisageable \\
\cline { 2 - 5 } & Forte & $\begin{array}{l}\text { Elle a trop de consé- } \\
\text { quences sur le revenu } \\
\text { des agriculteurs. Elle } \\
\text { est à éviter, sauf dans } \\
\text { la zone où il n'y a pas } \\
\text { de fortes variations de } \\
\text { revenu }\end{array}$ & $\begin{array}{l}\text { Elle peut résoudre le problème } \\
\text { précédent : les agriculteurs qui } \\
\text { gaspillent de l'eau sont pénali- } \\
\text { sés. Pour les autres, le revenu } \\
\text { ne se modifie pas }\end{array}$ & Envisageable \\
\hline
\end{tabular}

3) Elle doit prendre en compte le coît marginal social qui intègre les effets externes, comme la préservation des usages et de la qualité du milieu en aval.

4) Elle doit permettre l'équilibre budgétaire du gestionnaire et la maintenance des ouvrages collectifs.

5) Elle doit assurer une rentabilité sociale des investissements collectifs.

Dans le cas français, la première condition suffisante est toujours respectée, la deuxième presque jamais, la troisième est partiellement prise en compte, la quatrième est rencontrée et la cinquième envisagée.

D'autres instruments économiques peuvent être utilisés, en particulier si les conditions ne sont pas satisfaites. Une tarification reste toutefois nécessaire pour permettre au gestionnaire de couvrir ses coûts, mais elle ne sert alors plus à inciter les agriculteurs à avoir un comportement d'économie d'eau.

\subsection{Les quotas}

Le quota est un instrument qui, par nature, est contraignant et non incitatif.

Théoriquement, on peut montrer qu'une politique de quotas aboutit au même résultat qu'une politique tarifaire lorsque l'élasticité de la demande est infinie. D'autre part, en considérant la quantité d'eau disponible pour l'irrigation, une politique de quota est plus efficace qu'une politique de tarification, si la demande est inélastique et/ou si l'information n'est pas parfaite car, dans ce cas, une augmentation du prix de l'eau ne permettrait pas une diminution significative de la quantité consommée (Tableau 2).

\subsection{Les marchés de l'eau}

Ce système de gestion n'est pas utilisé en France et est ici introduit à titre de comparaison. C'est un mode d'allocation de la ressource en eau basé sur un échange de droits d'eau initialement attribués aux différents participants. Dans le cas d'un marché de l'eau entre agriculteurs, qui sont actuellement peu fréquents (Pakistan, Australie etc.), les offreurs peuvent utiliser l'eau pour leur usage personnel : ils arbitrent entre la valeur marginale de l'eau sur leur exploitation et le prix de vente du volume d'eau ou du droit d'eau établi sur le marché de l'eau. Cette valeur étant inférieure ou égale au prix de vente, il en résulte une valorisation accrue de la ressource en eau.

Pour qu'un marché de l'eau fonctionne de manière optimale, la théorie économique fournit un ensemble de conditions :

1) Conditions nécessaires:

- une ressource rare : pénurie de l'eau,

- un droit définissable, totalement spécifié, imposé et transférable,

- une organisation pré-existante ayant défini la répartition de droits d'eau (soit en terme de volume d'eau soit en terme de durée d'accès au système).

2) Conditions suffisantes:

- une possibilité d'échanger les droits d'accès à l'eau,

- un marché en situation de concurrence pure et parfaite (pas d'asymétries d'information ni de coûts de transaction),

- pas d'effets externes,

- pas de barrières sociologiques (acceptation par la société).

Ce système de gestion de l'eau, qui en pratique recouvre de multiples formes, est utilisé en particulier aux Etats-Unis Iorsque les droits d'eau ne correspondent plus à la réalité des usages du fait de l'évolution de l'environnement socio-économique.

Un exemple de marché de l'eau est fourni par les contrats d'option d'offre d'eau qui se pratiquent aux Etats-Unis entre les villes (non prioritaires en cas de sécheresse) et les agriculteurs. Ce sont des transferts temporaires d'eau d'irrigation pour sécuriser l'eau urbaine durant les sécheresses: ils garantissent la sécurité de l'offre d'eau urbaine pendant les périodes de sécheresse tout en maintenant la base de production agricole. En effet, l'option n'est exercée qu'en cas de nécessité ; lors des années climatiques normales, cette eau va à l'irrigation.

Les marchés de l'eau et la tarification sont deux instruments d'allocation différents que l'on peut comparer de la manière décrite dans le tableau 3.

Ainsi, le marché de l'eau est efficace pour répartir la ressource en eau à un niveau local étant donné que les lieux de décision sont décentralisés mais il pose le problème de la coordination à une plus grande échelle, car il ne permet pas, à lui seul, de prendre en compte les tiers. Une réglementation ou un système de taxes doivent alors être mises en place pour permettre aux marchés de l'eau de fonctionner efficacement. 
Tableau 3. - Comparaison du marché de l'eau et de la tarification.

\begin{tabular}{|l|l|l|}
\hline $\begin{array}{l}\text { Rareté de la } \\
\text { ressource } \\
\text { en eau }\end{array}$ & $\begin{array}{l}\text { Elle est prise en } \\
\text { compte dans les } \\
\text { droits d'eau }\end{array}$ & $\begin{array}{l}\text { Elle est annoncée } \\
\text { par la variation du } \\
\text { prix }\end{array}$ \\
\hline $\begin{array}{l}\text { Situation } \\
\text { du marché }\end{array}$ & $\begin{array}{l}\text { Concurrence } \\
\text { pure et parfaite }\end{array}$ & Monopole \\
\hline $\begin{array}{l}\text { Le droit } \\
\text { d'eau }\end{array}$ & $\begin{array}{l}\text { Le volume d'eau } \\
\text { ou la durée } \\
\text { d'accès sont } \\
\text { pré-définis }\end{array}$ & $\begin{array}{l}\text { La quantité d'eau est } \\
\text { variable d'une année } \\
\text { sur l'autre, selon le } \\
\text { contrat passé avec } \\
\text { le gestionnaire }\end{array}$ \\
\hline
\end{tabular}

\section{LA TARIFICATION ET LES QUO- TAS : DES INSTRUMENTS UTILISÉS EN FRANCE}

Deux instruments de gestion de l'eau d'irrigation sont utilisés en France pour inciter les agriculteurs à économiser de l'eau: la tarification et les quotas. Les exemples pris dans cet article ne sont qu'un échantillon pris parmi la diversité des situations constatées dans la pratique de la gestion de l'eau d'irrigation en France. Toutefois, il convient de préciser que les prix moyens qui sont donnés ne sont pas directement comparables, vu que ce ne sont pas les mêmes biens qui sont fournis aux agriculteurs : pour BRL (Bas-RhôneLanguedoc) et la SCP (Société du Canal de Provence), il s'agit d'une eau sous pression à la borne d'irrigation, tandis que pour la Charente et la CACG (Compagnie des Coteaux de Gascogne), il s'agit de l'eau à la rivière. L'utilisateur doit alors ensuite en assurer le pompage et la mise en pression.

\subsection{La tarification}

Les principes étant respectés, la tarification peut être conçue dans une optique de demande ou dans une optique d'offre, même si, en pratique, les tarifs établis conjuguent les deux aspects.

\section{I.I Tarification selon la demande}

Dans une optique de demande, il est possible de tarifer: - en fonction des usages : soit on discrimine les prix, selon la nature des cultures par exemple, en vue de récupérer une partie du surplus lié à l'irrigation, soit on met en concurrence les différents usages et on attribue l'eau en fonction des utilités marginales;

- en prenant en compte des contraintes d'équité.

Prenons le cas de BRL qui est une Société d'Aménagement Régional (SAR) située sur la façade méditerranéenne entre le Rhône et l'Espagne.

Les SAR (BRL, CACG, SCP) ont été créées en France entre 1955 et 1960 sous l'égide du Ministère de l'Agriculture; elles ont en particulier pour objet la mise en valeur agricole, plus spécialement par l'exécution et l'exploitation de grands ouvrages hydrauliques pour l'irrigation, de régions où des conditions physiques, climatiques, économiques ou sociales avaient retardé l'évolution et l'adaptation par rapport au reste de la France. Ces sociétés d'économie mixte sont concessionnaires d'Etat et exercent à ce titre une mission de service public. Elles sont administrées comme des sociétés anonymes de droit privé mais qui bénéficient de prérogatives réservées aux entreprises publiques et de moyens de financement publics. Le capital social est détenu en majorité par des collectivités publiques.

La tarification de BRL est principalement tournée vers les agriculteurs (130000 hectares) qui représentent $70 \%$ des volumes consommés et $50 \%$ des recettes. Elle a essayé d'établir une tarification en fonction de la demande, sous contrainte d'un équilibre budgétaire de plus en plus réel depuis la disparition de la prise en charge des charges intercalaires par l'Etat.

Cela l'a conduit en 1992 à réviser son tarif, en vu d'augmenter ses recettes de $20 \%$. Les trois principes sur lesquels repose la tarification sont :

- la péréquation : tous les agriculteurs paient le même prix, sauf en cas de pompage supplémentaire. Il n'y a pas de distinction par zone ;

- la prise en compte des caractéristiques de la demande par un choix entre différents contrats et différents tarifs ne comprenant pas de volume forfaitaire. En particulier, l'irrigant peut choisir entre un «tarif pro», qui se caractérise par une redevance débit élevée mais une redevance au volume faible, et le «tarif appoint », où c'est l'inverse. Ce dernier tarif est interressant pour les cultures qui ne sont irriguées que les années particulièrement sèches

- une prise en compte des coûts : les tarifs les moins élevés se situent au-dessus du coût marginal.

Sachant qu'il existe des écarts de prix très importants, on peut donner un indice moyen des différents prix de l'eau pratiqués en 1993 :

Irrigation agricole : $0,95 \mathrm{~F} / \mathrm{m}^{3}$.

Eau brute : $1,70 \mathrm{~F} / \mathrm{m}^{3}$.

Eau potabilisée : $2,70 \mathrm{~F} / \mathrm{m}^{3}$.

Usages divers : $3,50 \mathrm{~F} / \mathrm{m}^{3}$.

\subsubsection{Tarification selon l'offre}

Dans une optique d'offre, deux sortes de coûts entrent en jeu: - il y a des coûts qui sont liés à l'amenée de l'eau à la parcelle. Ils sont supportés en grande partie par le gestionnaire. L'organisme gestionnaire peut envisager deux modes de tarification : une tarification en fonction du coût moyen ou du coût marginal ;

- mais il y a aussi des coûts qui ne sont pas pris en compte par le gestionnaire : en effet, il existe des coûts qui sont supportés par l'usager mais aussi des effets externes négatifs (pollution, encombrement, durabilité) qui sont subis par la collectivité et qui sont à l'origine de coûts sociaux. Dans un système tarifaire, une approche du point de vue de la collectivité, c'est-à-dire du point de vue de l'ensemble des usages, y compris le maintien de la qualité de l'hydrosystème, est nécessaire, ce qui est l'objectif des agences de l'eau.

Pour la gestion de l'eau dans une optique de l'offre, nous nous proposons de distinguer deux cas représentatifs selon que la ressource en eau n'est pas contraignante ou qu'elle est limitée : la SCP et la Charente.

\subsubsection{1. le cas d'une ressource non contraignante : la SCP}

La SCP est située au sud-est de la France, dans la région Provence-Alpes-Côte d'Azur. Du fait des aménagements réalisés (réseaux d'amenée d'eau et barrages), la ressource en eau n'est pas contraignante en période d'étiage et assure l'irrigation d'environ 70000 ha.

Une tarification au coût marginal de long terme a été mise en ouvre par la SCP. Elle consiste à inclure non seulement un terme relatif au coût variable mais aussi un autre terme permettant l'extension des capacités de production et assurant la pérennité de l'entreprise. Conformément à la théorie économique, elle vise à obtenir une situation Pareto-optimale, c'est-à-dire optimale du point de vue de la collectivité.

Pratiquement, cette grandeur économique est approximée par la notion de coût de développement qui est calculé sur la base de la somme des valeurs actualisées des dépenses éche- 
lonnées dans le temps faites pour permettre de satisfaire les besoins présents et futurs des utilisateurs.

La tarification de la SCP se base sur la théorie économique mais elle a été amenée à négocier les tarifs par usagers en fonction de considérations économiques et sociales (aide à une catégorie d'usagers) ou de considérations géographiques (éloignement de la ressource). Ainsi le prix de l'eau d'irrigation pour l'agriculture est fixé à $40 \%$ du coût de développement.

Les tarifs sont différenciés selon:

- les catégories d'usagers (consommateurs individuels d'eau d'irrigation à usage agricole, consommateurs individuels d'eau à usages divers, collectivités consommatrices d'eau livrée en gros, industriels etc...),

- I'implantation des usagers par rapport à l'origine de la ressource (zones tarifaires),

- l'époque à laquelle les demandes d'eau se manifestent (tarifs saisonniers),

- Ies demandes particulières des clients.

La SCP propose différents tarifs. Pour un usage normal, ils comportent trois termes:

1) une redevance annuelle proportionnelle au débit souscrit par l'abonné incluant dans certains cas une redevance forfaitaire de consommation pour les prises ayant fait l'objet d'un contrat d'eau d'irrigation agricole (volume forfaitaire de 100 mètres cubes par mètre cube/heure souscrit). Elle est assise sur les coûts des investissements ;

2) une redevance de consommation annuelle : la consommation d'eau est facturée au mètre cube ; la redevance est différente selon que l'eau est consommée pendant les périodes de pointe (15 mai-14 septembre) ou hors pointe, sauf pour les consommations agricoles;

3) une redevance supplémentaire de pompage, correspondant au prix de l'énergie, quand la Société est contrainte d'effectuer un relèvement des eaux pour desservir l'abonné.

Un terme supplémentaire peut être ajouté en cas de traitement assuré par la SCP (potabilisation-clarification).

Connaissant les limites de la notion de prix moyen, on peut toutefois donner un indice moyen des différents prix de l'eau pratiqués en 1993 :

Tous usages confondus : $1,75 \mathrm{~F} / \mathrm{m}^{3}$.

Par usage :

Irrigation agricole : $0,61 \mathrm{~F} / \mathrm{m}^{3}$.

Irrigation non agricole : $2,16 \mathrm{~F} / \mathrm{m}^{3}$.

Usages divers : $4,95 \mathrm{~F} / \mathrm{m}^{3}$.

Eaux urbaines : $1,99 \mathrm{~F} / \mathrm{m}^{3}$.

Eaux industrielles : $2,01 \mathrm{~F} / \mathrm{m}^{3}$.

\subsubsection{2 le cas d'une ressource en eau limitée: la Charente}

Le cas présenté résulte d'une étude en cours réalisée par le Cemagref à la demande du Conseil Général de la Charente et ne peut être considéré comme un cas concret de gestion de l'eau.

On se situe dans un contexte de déséquilibre entre le débit de la Charente d'une part, le respect des débits d'étiage et la demande des usagers (irrigation, alimentation en eau potable) d'autre part. Pour améliorer l'offre en eau, la construction d'un nouveau barrage a été décidée.

Le futur plan de gestion d'étiage sera plus contraignant que celui en vigueur à l'heure actuelle et une tarification sera mise en place.

Le dispositif retenu comporte les éléments suivants:

a) une part fixe de $75 \mathrm{~F}$ par hectare irrigué,

b) une part variable proportionnelle au volume consommé sur la base d'un tarif de référence de $0,0375 \mathrm{~F} / \mathrm{m}^{3}$,

c) une pénalité par multiplication de ce tarif de référence par 10 pour les volumes utilisés au-delà des seuils de référence,

d) une actualisation du tarif de référence fondé sur l'évolution de la somme de la valeur marchande du produit d'un hectare (composé de $90 \%$ de maïs et de $10 \%$ de pois protéagineux) et de la prime compensatoire «PAC (Politique Agricole Commune),

e) une indexation de cet ensemble tarifaire fonction du taux de remplissage du barrage actuel de Lavaud et du barrage futur de Mas-Chaban.

Pour analyser l'impact de la tarification de l'eau dans le bassin de la Charente sur le comportement des agriculteurs, des simulations économiques ont permis d'établir des courbes de demande et d'en déduire la sensibilité de la consommation de l'eau par rapport au prix de l'eau d'irrigation.

La taille de l'échantillon (170 exploitations irrigantes) et la précision des données mobilisées confèrent aux résultats présentés une représentativité des régions de grandes cultures.

La valorisation de l'irrigation est de $0,96 \mathrm{~F} / \mathrm{m}^{3}$ en moyenne sans tenir compte de la rémunération des facteurs fixes spécifiques (main d'œuvre permanente et matériel d'irrigation).

Le supplément de revenu procuré par l'irrigation représente une part importante du revenu total et excède le niveau des primes spécifiques PAC aux cultures irriguées ( $1415 \mathrm{~F} / \mathrm{ha}$ en 1993 pour le maïs). Il explique bien le niveau

Tableau 4. - Critères de rentabilité privée des exploitations de grandes cultures irrigantes.

\begin{tabular}{|c|c|c|c|c|c|c|}
\hline Type & $\begin{array}{c}\text { SAU } \\
\text { moyenne } \\
\text { (hectares) } \\
\% \text { de SI }\end{array}$ & $\begin{array}{l}\text { Cultures } \\
\text { irriguées }\end{array}$ & $\begin{array}{c}\text { Augmentation } \\
\text { du revenu } \\
\text { moyen }\end{array}$ & $\begin{array}{c}\text { Part } \\
\text { dans le revenu } \\
\text { total }(\%)\end{array}$ & $\begin{array}{l}\text { Valorisation } \\
\text { de I'eau } \\
\text { en } \mathrm{F} / \mathrm{m}^{3}\end{array}$ & $\begin{array}{l}\text { Valeurs** } \\
\text { marginales } \\
\text { de l'eau } \\
\text { en } \mathrm{F} / \mathrm{m}^{3}\end{array}$ \\
\hline $\begin{array}{l}\text { Petit irrigant } \\
\text { céréalier }\end{array}$ & $\begin{array}{l}72 \\
15\end{array}$ & $\begin{array}{l}\text { Maïs }(82 \%) \\
\text { Pois }(10 \%) \\
\text { Tournesol }(8 \%)\end{array}$ & 17000 & 26 & 0,95 & de 0 à 2,49 \\
\hline $\begin{array}{l}\text { Céréalier irrigant } \\
\text { maïsiculteur }\end{array}$ & $\begin{array}{l}75 \\
37\end{array}$ & $\begin{array}{l}\text { Mais }(87 \%) \\
\text { Pois }(13 \%)\end{array}$ & 41200 & 41 & 1,00 & de 0 à 1,21 \\
\hline Maïsiculteur & $\begin{array}{l}92 \\
72\end{array}$ & $\begin{array}{l}\text { Maïs }(90 \%) \\
\text { Pois }(10 \%)\end{array}$ & 116000 & 66 & 1,04 & de 0 à 1,21 \\
\hline $\begin{array}{l}\text { Céréalier et } \\
\text { diversification des } \\
\text { cultures irriguées }\end{array}$ & $\begin{array}{l}67 \\
54\end{array}$ & $\begin{array}{l}\text { Maïs }(62 \%) \\
\text { Pois }(13 \%) \\
\text { Tournesol }(25 \%)\end{array}$ & 55000 & 76 & 0,83 & 0,88 à 2,32 \\
\hline
\end{tabular}

* La valeur marginale de l'eau est fortement dépendante de l'état de la ressource. Nous avons choisi en conséquence de fournir les plages de variation rencontrées en fonction des différents niveaux de débit d'étiage respectés dans le fleuve.

SAU = Surface Agricole Utile $; S \mid=$ Surface Irriguée.

Source: RIEU et PALACIO, 1994. 


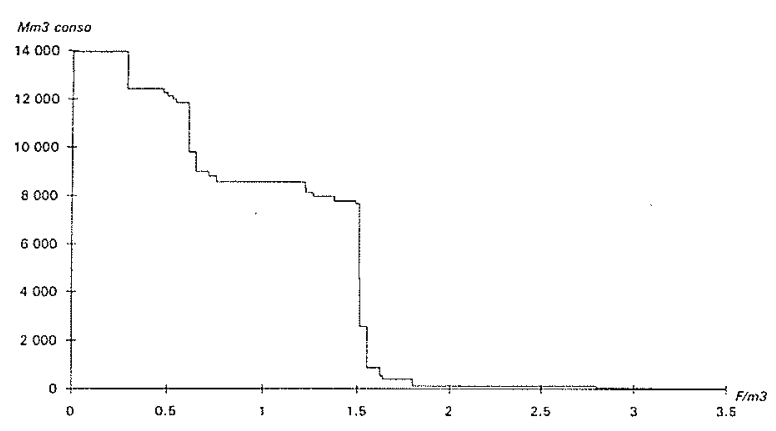

3. Courbe de demande en eau.

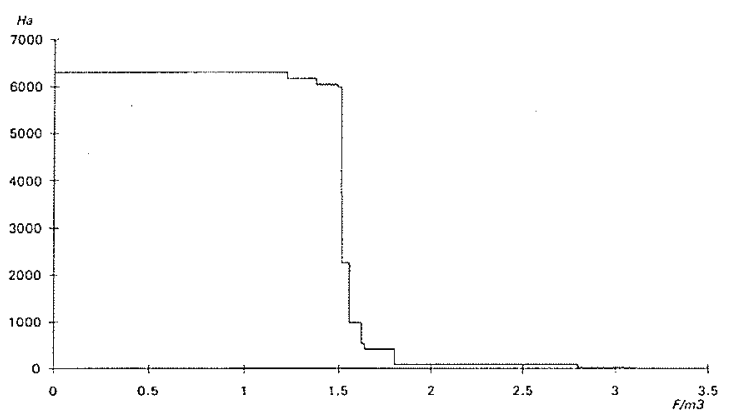

4. Surface irriguée en fonction du prix.

de la demande et le développement récent de l'irrigation en grandes cultures.

La demande en eau est sensible au prix. Elle n'est pas linéaire. L'élasticité de la demande est forte au voisinage de $1,51 \mathrm{~F} / \mathrm{m}^{3}$. A ce prix, on observe une diminution importante de la quantité d'eau consommée qui s'accompagne d'une restriction significative des surfaces irriguées, ce qui ne se passait pas lors du précédent saut (pour un prix de $0,61 \mathrm{~F} / \mathrm{m}^{3}$ ) où on avait une diminution de l'apport d'eau aux plantes. Au delà d'un prix de $1,55 \mathrm{~F} / \mathrm{m}^{3}$, la demande en eau est pratiquement inexistante.

En moyenne, la surface irriguée dans une exploitation couvre $38 \%$ de la SAU. Elle chute de $6 \%$ lorsqu'on atteint un prix de $1,55 \mathrm{~F} / \mathrm{m}^{3}$. Ce pourcentage cache de fortes disparités en fonction du type d'exploitations : une exploitation tournée vers l'élevage consacre moins de surface à l'irrigation qu'une exploitation tournée vers la culture du maïs.

L'étude de la sensibilité de la demande en eau d'irrigation par rapport au prix doit être complétée par une étude de la sensibilité du revenu. Cela permet d'éviter de préconiser une augmentation trop forte du prix de l'eau pour inciter les agriculteurs à économiser l'eau qui ne serait pas acceptable en terme de revenu. En considérant un revenu minimum de 70000 francs, la partie proportionnelle du prix de l'eau d'irrigation ne devrait pas dépasser $1,27 \mathrm{~F} / \mathrm{m}^{3}$.

Par ailleurs, ces résultats sont à nuancer en tenant compte des hétérogénéités de revenu entre les exploitations.

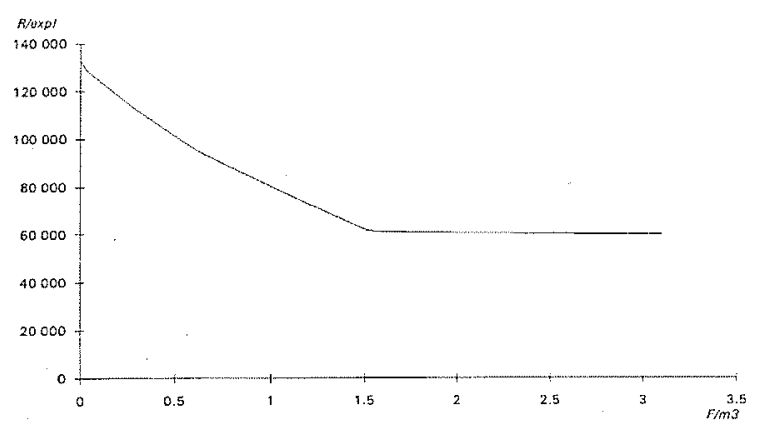

5. Courbe de revenu par exploitation.
Cette étude nous permet de constater que le prix proposé lors du projet de barrage n'incitera pas les agriculteurs à avoir un comportement économe en eau, vu qu'ils ne sont pas sensibles à ce prix $\left(0,0375 \mathrm{~F} / \mathrm{m}^{3}\right)$. De plus, le tarif considéré comme dissuasif (décuplement du prix de l'eau, soit $0,375 \mathrm{~F} / \mathrm{m}^{3}$ ) ne l'est pas : lorsque le prix de l'eau passe de 0,0375 à $0,375 \mathrm{~F} / \mathrm{m}^{3}$, le comportement des agriculteurs ne se modifie pas. Toutefois, c'est une étude qu'il faut soumettre à l'expérience pour voir le comportement réel des agriculteurs une fois le barrage construit.

Ainsi, l'instauration d'une tarification est un processus complexe dont le résultat, à savoir la structure tarifaire, doit rester simple, compréhensible et peu coûteux.

\subsection{Les quotas}

\subsection{Une politique de quota : le cas de la Charente}

En Charente, la politique de quota se traduit par le plan de gestion d'étiage. A cette période, les agriculteurs n'ont pas le droit d'irriguer le dimanche et des restrictions progressives sont prévues en cas de débit inférieur à des seuils prédéfinis.

Tableau 5. - Plan de gestion d'étiage du département de la Charente en 1995.

\begin{tabular}{|l|l|l|}
\hline $\begin{array}{c}\text { Seuil } \\
\text { d'alerte }\end{array}$ & $\begin{array}{l}\text { Débit à } \\
\text { l'étiage }\end{array}$ & \multicolumn{1}{|c|}{ Mesures correspondantes } \\
\hline Préalerte & $4 \mathrm{~m}^{3} / \mathrm{s}$ & $\begin{array}{l}\text { interdiction des prélèvements } \\
\text { 2 jours par semaine }\end{array}$ \\
\hline Alerte 1 & $2,5 \mathrm{~m}^{3} / \mathrm{s}$ & $\begin{array}{l}\text { interdiction des prélèvements } \\
\text { 3 jours par semaine }\end{array}$ \\
\hline Alerte 2 & $1,8 \mathrm{~m}^{3} / \mathrm{s}$ & $\begin{array}{l}\text { interdiction des prélèvements } \\
5 \text { jours par semaine }\end{array}$ \\
\hline Alerte 3 & $1,2 \mathrm{~m}^{3} / \mathrm{s}$ & interdiction totale des prélèvements \\
\hline
\end{tabular}

Ces mesures peuvent être facilement détournées dans les premiers seuils d'alerte par les agriculteurs sur-équipés en matériel d'irrigation: ils peuvent irriguer davantage les autres jours de la semaine, pour compenser les jours d'interdiction.

\subsubsection{Quota et tarification : une application simulta-} née

Une solution consiste à combiner les deux systèmes : elle est appliquée en France par la CACG dans le système Neste dès 1990.

La CACG est une SAR implantée dans le sud-ouest de la France. Son système de distribution est très différent de BRL et de la SCP. Pour la distribution de l'eau et son stockage, elle utilise le système des barrages et des rivières réalimentées dans lesquelles prélèvent les agriculteurs. Cette solution technique permet de minimiser les couts de transport de l'eau. En contre partie et du fait de ressources limitées, elle doit mettre en place un système de gestion efficace qui évite des prélèvements trop élevés. Le dispositif choisi combine une police des eaux et une tarification.

La tarification a évolué depuis les années 60: au départ, pour encourager l'irrigation, la tarification mise en auvre était de type monôme dégressive. Durant les années 70 , on souhaite densifier les réseaux en établissant une tarification binôme dégressive (qui encourage les consommateurs les plus importants) mais qui ne prend pas en charge les frais d'investissement. Depuis 1978 et la disparition des aides 
publiques, la tarification binôme prend en charge les frais de renouvellement des installations.

Un système spécifique a été élaboré pour les contrats individuels et les Associations Syndicales Autorisées. C'est le système Neste qui instaure un système de quota de $4000 \mathrm{~m}^{3} \mathrm{par} 1 / \mathrm{s}$ souscrit sur les rivières réalimentées en 1989. Si les agriculteurs dépassent le quota annuel, le prix $\mathrm{du} \mathrm{m}^{3}$ supplémentaire est de $0,12 \mathrm{p}(\mathrm{p}=5,08 \mathrm{~F}$ en 1991, soit $\left.0,61 \mathrm{~F} / \mathrm{m}^{3}\right)$. Ce prix est considéré comme dissuasif. Si les ressources ne sont pas suffisantes, le quota est diminué avec partage de la perte entre la CACG et l'usager.

La gestion par les quotas a l'avantage d'offrir de solides garanties quant au résultat final. Il peut même tenir compte de la probabilité de défaillance de la ressource : le quota est garanti 8 années sur 10 et un quota de $20 \%$ inférieur est garanti 2 années sur 10 à la CACG. Toutefois, ils sont lourds à mettre en place; de plus, ils ont souvent un caractère rigide (on ne peut pas les modifier chaque année) et non incitatif.

\section{$\mathrm{V}$ CONCLUSION}

En France, dans le domaine de l'eau agricole, tous les types d'instruments économiques sont présents, à l'exception des marchés de l'eau. Ils sont mis en œuvre en fonction des conditions de mobilisation de la ressource et des caractéristiques de la demande. On rencontre ainsi partout:

- le prélèvement de redevances par les agences de bassin, sur la base du principe préleveur-payeur mais avec un niveau sensiblement inférieur au coût d'opportunité de l'eau dans son site et avec des exonérations sous certaines conditions ;

- une tarification dont les objectifs peuvent être l'équilibre budgétaire du gestionnaire, la participation aux investissements, la durabilité des ouvrages et des incitations à économiser l'eau.

Les tarifications observées reposent le plus souvent sur une tarification au coût moyen. La compagnie du BasRhône-Languedoc prend particulièrement en compte la demande des usagers, la Compagnie d'Aménagement des Coteaux de Gascogne applique un système combinant quotas et tarification, enfin la Société du Canal de Provence utilise la tarification de l'eau au coût marginal. L'application de ce principe fournit un cadre théorique et par suite un outil de négociation robuste avec les usagers.

Le caractère incitatif de la tarification varie beaucoup en fonction de l'importance de la part proportionnelle dans le tarif et de l'élasticité de la demande au prix. Ainsi la demande peut être sensible au prix de l'eau en grandes cultures où le prix de l'eau représente une part importante des charges d'exploitation de l'agriculteur, alors qu'elle le. sera rarement pour des cultures à haute valeur ajoutée. Dans ce dernier cas, d'autres instruments sont mis en place comme les quotas.

Les redevances prélevées par les agences de bassin devraient refléter la concurrence entre les usages dont le maintien de la qualité de l'hydrosystème, mais en pratique, elles affectent peu le comportement des irrigants car leur montant est peu important par rapport aux autres compo- santes du coût de l'irrigation pour l'agriculteur et n'influent pas généralement sur la demande.

La mise en application de la Loi sur l'eau, qui affirme l'unicité de la ressource en eau, qui impose des dispositifs de comptage et qui donne un cadre de négociation à travers des lieux de concertation (Commissions Locales de l'Eau) et des Schémas Directeurs d'Aménagement de Gestion des Eaux (SDAGE), renouvelle l'intérêt pour les instruments incitatifs de gestion, leurs conditions de mise en ouvre et leurs limites.

\section{Remerciements}

Les auteurs remercient J. Astier, J. Cothenet, M. Jean, B. Lesaffre, Y. Mérillon, M. Mesny, J.P. Nicol, V. Palacio, J. Plantey, L. Rieul et $\mathrm{H}$. Tardieu pour leurs commentaires et leurs suggestions.

\section{BIBLIOGRAPHIE}

11] Hurand P. (1994.). - Water management in the Neste system. Kluwer Academic Publishers.

[2] JEAN M. (1980). - L'application de la théorie du coût marginal au tarif de vente d'eau dans un ouvrage à buts multiples. Document interne à la SCP.

|3| Journal Offíciel de la République Française 1992. Loi n $92-$ 3 janvier 1992 sur l'eau, parue au JORF le 4 janvier 1992.

[4] Micheisen A et Young R. (1993). - Optioning agricultural water rights for urban water supplies during drought. American Journal of Agricultural Economic : 1010-1020.

[5] MusEau (groupe de travail) (1994). - La valeur de l'eau d'irrigation, application aux coteaux du Gers, Synthèse, 16 mars.

[6] Nicolazo J.L. (1994), - Les agences de l'eau, P. Johanet, Paris.

[7] PALACIO V. et RIEU T. (1994). - Equipements hydrauliques collectifs et réforme de la PAC: des conséquences conflictuelles ? Le cas d'un projet de barrage en Charente. INRA-ESR. Actes et communications. Vol. $12:$ 185-203.

181 PAOLI D. et Rieu T. (1992). - La situation de l'eau en France. Economie et Statistique. Vol. 258-259.

[9] Rieu T. et Strosser P. (1993). - A research methodology to analyse the impact of water markets on quality of irrigation services and agricultural production. IIMI, Pakistan.

[10] Société du Canal de Provence (1989). - Livre bleu sur les prix et tarif de l'eau brute urbaine du Canal de Provence. Document interne. Septembre.

[11] Tardieu H. (1992). - La CACG, organe de gestion de l'eau: un instrument technique et un lieu de concertation. La Houille Blanche. 